\title{
Inhibition of miR-483-5p improves the proliferation, invasion and inflammatory response of triple-negative breast cancer cells by targeting SOCS3
}

\author{
JIANBO REN ${ }^{1 *}$, GANG XU $^{2 *}$, HONGYAN SUN ${ }^{3}$, TING LIN $^{4}$, SANHUI XU ${ }^{5}$ and YATING ZHAO ${ }^{6}$ \\ ${ }^{1}$ Department of Oncology, Yantai Muping Hospital of Traditional Chinese Medicine, Yantai, Shandong 264100; \\ ${ }^{2}$ Key Laboratory of Carcinogenesis and Translational Research (Ministry of Education/Beijing), \\ Department of Radiation Oncology, Peking University Cancer Hospital and Institute, Beijing 100142; \\ ${ }^{3}$ Endoscopy Center, Yantai Muping Hospital of Traditional Chinese Medicine, Yantai, Shandong 264100; \\ ${ }^{4}$ Department of Breast Surgery, Tangshan People's Hospital, Lunan, Tangshan, Hebei 063000; \\ ${ }^{5} 1$ st Laboratory Department, Xingtai People's Hospital, Xingtai, Hebei 054000; ${ }^{6}$ Breast Health Department, \\ Tangshan Maternal Child Health Care Hospital, Tangshan, Hebei 063000, P.R. China
}

Received April 20, 2020; Accepted April 21, 2021

DOI: $10.3892 / \mathrm{etm} .2021 .10480$

\begin{abstract}
RNAs (miRs) have been indicated to serve oncogenic or tumor suppressor roles. However, the role of miR-483-5p in breast cancer and its associated molecular mechanisms remain unclear. In the present study, compared with adjacent normal tissues and MCF-10a cells, the expression level of miR-483-5p was upregulated in triple-negative breast cancer (TNBC) tissues and TNBC cell lines. Bioinformatic analysis and luciferase reporter assay confirmed the presence of miR-483-5p binding sites in the 3'-untranslated region of suppressor of cytokine signaling 3 (SOCS3). In addition, the expression level of SOCS3 protein in TNBC tissues was markedly lower compared with in adjacent tissues, and miR-483-5p expression was negatively correlated with SOCS3 expression in TNBC tissues. Cell proliferation and flow cytometry assays indicated that knockdown of miR-483-5p inhibited the proliferation and promoted apoptosis in the TNBC cell line BT-549. This effect was markedly attenuated by SOCS3 small interfering (si)RNA transfection. Additionally, wound healing and Transwell assays demonstrated that SOCS3 siRNA reversed the inhibitory effects of miR-483-5p inhibitor on the migration and invasion of BT-549 cells. Moreover, the decrease in miR-483-5p expression significantly reduced the secretion of TNF- $\alpha$, IL- 6 , IL-1 $\beta$ and monocyte chemoattractant protein-1 in BT-549 cells, while SOCS3 siRNA could partially reverse
\end{abstract}

Correspondence to: Dr Yating Zhao, Breast Health Department, Tangshan Maternal Child Health Care Hospital, 14 Jianshe South Road, Tangshan, Hebei 063000, P.R. China

E-mail: zhaoyating1977@webmailcn.com

*Contributed equally

Key words: triple negative breast cancer, microRNA-483-5p, inflammatory response, suppressor of cytokine signaling 3 this effect. Additionally, SOCS3 overexpression reversed the effects of miR-483-5p mimic on the proliferation, migration, invasion and inflammation of BT-549 cells. Taken together, these data demonstrated that the inhibition of miR-483-5p could inhibit the proliferation, migration, invasion and inflammatory response, while promoting the apoptosis of TNBC cells by negatively regulating SOCS3. miR-483-5p may be a potential target for TNBC therapy.

\section{Introduction}

Breast cancer is the leading cause of cancer-associated mortality in women worldwide (1). Triple-negative breast cancer (TNBC) is a special subtype of breast cancer, accounting for $\sim 10 \%$ of all breast cancers (2). The clinical manifestations of TNBC include high invasive capacity, tendency for early metastasis and a lack of recognizable therapeutic targets, which is directly associated with the poor clinical prognosis of TNBC (3). Identification of relevant biological markers could provide novel strategies for the diagnosis and treatment of TNBC.

MicroRNAs (miRNAs/miRs) are a type of single-stranded non-coding small RNA with a length of 20 nucleotides (4). It has been indicated that miRNAs serve important regulatory roles in development, cell differentiation, proliferation, apoptosis and tumor invasion, metastasis and the tumor microenvironment (5). miRNAs specifically bind to the 3'-untranslated region (3'-UTR) of their downstream target gene mRNA to promote mRNA degradation or inhibit mRNA translation (4). It has been indicated that the abnormal expression of miRNAs is closely associated with the occurrence and progression of several malignant tumors. For instance, miR-183 has been revealed to target sirtuin 1 or the PI3K/AKT/mTOR signaling pathway to regulate the apoptosis and autophagy of gastric cancer cells (6). miR-488 has been demonstrated to inhibit the apoptosis of $\mathrm{CD}^{+} \mathrm{T}$ cells in colon cancer through the inhibition of indoleamine 2,3-dioxygenase 1, which may be beneficial for the development of novel immunotherapy 
drugs for colon cancer (7). Upregulation and downregulation of multiple miRNAs has also been demonstrated to exist in breast cancer. For example, the miR-10 family (8), miR-21 (9) and the miR-17/92 cluster (10) are considered to serve a role in promoting cancer, while the let-7 (11) and miR-200 families (12) are considered as inhibitors in breast cancer. Localized at 11p15.5, miR-483-5p is located in the second intron of insulin-like growth factor 2 (13). miR-483-5p has been revealed to be dysregulated and has been associated with poorer survival in adrenocortical carcinoma and lung adenocarcinoma $(14,15)$. However, the role of miR-483-5p in breast cancer and its associated molecular mechanisms remain unclear.

The present study measured the expression of miR-483-5p in TNBC to investigate its role and the underlying molecular mechanism.

\section{Materials and methods}

Clinical samples. Tumor and adjacent tissues ( $>5 \mathrm{~cm}$ away from tumor tissues) of 25 female patients with TNBC who underwent surgical resection in Tangshan Maternal Child Health Care Hospital (Tangshan, China) between October 2018 and October 2019 were collected. The age range of the patients was $29-68$ years, and the mean age was $42 \pm 1.9$ years. None of the patients received any treatment before surgery. Immediately after the excision, the tissues were immersed in liquid nitrogen and stored at $-80^{\circ} \mathrm{C}$. The present study was approved by Yantai Muping Hospital of Traditional Chinese Medicine (Yantai, China), and written informed consent was obtained from all the participants.

Cell culture. The non-malignant breast epithelial cell line MCF-10a and TNBC cell lines (BT-20, MDA-MB-231, MDA-MB-468 and BT-549) were obtained from American Type Culture Collection (ATCC) and maintained in RPMI-1640 medium (HyClone; Cytiva) containing 10\% FBS (Gibco; Thermo Fisher Scientific, Inc.) and $1 \%$ penicillin-streptomycin (Invitrogen; Thermo Fisher Scientific, Inc.) at $37^{\circ} \mathrm{C}$ in a humidified atmosphere with $5 \% \mathrm{CO}_{2} .293 \mathrm{~T}$ cells were obtained from ATCC and cultured in high-glucose DMEM (HyClone; Cytiva) containing $10 \% \mathrm{FBS}$, at $37^{\circ} \mathrm{C}$ in a $5 \% \mathrm{CO}_{2}$ incubator.

Reverse transcription-quantitative PCR (RT-qPCR). Total RNA was extracted from BT-549 cells using an RNeasy Mini kit (Qiagen $\mathrm{GmbH}$ ). cDNA was synthesized from 500 ng RNA using PrimeScript RT Master Mix (cat. no. RR036Q; Takara Biotechnology Co., Ltd.) according to the manufacturer's protocol. Total RNA was reverse-transcribed into mature miRNAs using a Mir-X miRNA First-Strand Synthesis kit (Takara Biotechnology Co., Ltd.) according to the manufacturer's protocol. SOCS3 expression was measured using SYBR-Green RT-qPCR kit (Qiagen $\mathrm{GmbH}$ ). The reactions were carried out in a Veriti Thermal Cycler (Applied Biosystems; Thermo Fisher Scientific, Inc.) following the cycling: $50^{\circ} \mathrm{C}$ For $30 \mathrm{~min}, 95^{\circ} \mathrm{C}$ for $15 \mathrm{~min}$, followed by 45 cycles of $94^{\circ} \mathrm{C}$ for $30 \mathrm{sec}, 58^{\circ} \mathrm{C}$ for $30 \mathrm{sec}, 72^{\circ} \mathrm{C}$ for $30 \mathrm{sec}$ and a final extension of $72^{\circ} \mathrm{C}$ for $10 \mathrm{~min}$. miR-483-5p expression was detected with a Hairpin- $\mathrm{it}^{\mathrm{TM}}$ miRNAs qPCR kit (Shanghai GenePharma Co.,
Ltd.). The reactions were carried in Veriti Thermal Cycler following the cycling: $95^{\circ} \mathrm{C}$ For 3 min followed by 40 cycles of $95^{\circ} \mathrm{C}$ for $12 \mathrm{sec}, 58^{\circ} \mathrm{C}$ for $30 \mathrm{sec}$ and a final extension of $72^{\circ} \mathrm{C}$ for $10 \mathrm{~min}$. Quantitative analysis was performed using the $2^{-\Delta \Delta \mathrm{Cq}}$ method (16). U6 and GAPDH were used as endogenous normalization controls for miRNA and mRNA, respectively. The primers used are listed as follows: miR-483-5p forward, 5'-AGT TGGCTCACGGTTCTTTCAA-3' and reverse, 5'-ATCGCCA TGGCCCGCATGTCGG-3'; SOCS3 forward, 5'-AGAGCGG ATTCTACTGGAGCG-3' and reverse, 5'-CTGGATGCGTAGG TTCTTGGTC-3'; U6 forward, 5'-CTCGCTTCGGCAGC ACA-3' and reverse, 5'-AACGCTTCACGAATTTGCGT-3'; GAPDH forward, 5'-ATGTTGCAACCGGGAAGGAA-3' and reverse, 5'-GCAAATTCGTGAAGCGTTCCATA-3'.

Cell transfection. miR-483-5p mimics, miR-483-5p inhibitor, small interfering (si)RNA targeting SOCS3 (si-SOCS3; forward, 5'-UAGGAGACUCGCCUUAAAUTT-3'; reverse, 5'-AUUUA AGGCGAGUCUCCUATT-3') and non-targeting sequence (si-Ctrl; forward, 5'-UUCUCCGAACGUGUCACGUTT-3' and reverse, 5'-ACGUGACACGUUCGGAGAATT-3') were purchased from Shanghai GeneChem Co., Ltd. SOCS3 overexpression vector (SOCS3-OE; forward, 5'-AAAGCTAGCCC ATGGTCACCCACAGCAAGTT-3' and reverse, 5'-AAACTC GAGTCCTTAAAGTGGAGCATCATAC-3') and empty vector (pcDNA3.1) were obtained from Invitrogen (Thermo Fisher Scientific, Inc.). BT-549 cells were seeded at $1.5 \times 10^{5}$ cells/well in a six-well plate and cultured for $24 \mathrm{~h}$. The cells were transiently transfected $24 \mathrm{~h}$ post-seeding with mimics $(50 \mathrm{pmol} / \mathrm{ml})$, inhibitors $(50 \mathrm{pmol} / \mathrm{ml})$, si-SOCS3 $(50 \mathrm{nM})$ or SOCS3-OE $(2 \mu \mathrm{g})$ using Lipofectamine 2000 (Invitrogen; Thermo Fisher Scientific, Inc.) according to the manufacturer's instructions.

Dual-luciferase reporter assay. To determine if SOCS3 is a target of miR-483-5p, the target gene prediction software miRTarBase (https://mirtarbase.cuhk.edu.cn/ miRTarBase/ miRTarBase_2019/php/index.php) was used. Wild-type (Wt) and mutant (Mut) SOCS3 3'-UTRs were amplified and cloned into the luciferase reporter vector pmirGLO (Promega Corporation). BT-549 and 293T cells from the miR-negative control (NC) and miR-483-5p groups were seeded in 24-well plates (1x10 4 cells/well), and transfected with SOCS3-Wt or SOCS3-Mut plasmids $(0.5 \mu \mathrm{g})$, respectively, using Lipofectamine ${ }^{\mathrm{TM}} 2000$ (Invitrogen; Thermo Fisher Scientific, Inc.), and cultured for $48 \mathrm{~h}$ at $37^{\circ} \mathrm{C}$. After $48 \mathrm{~h}$ of incubation, cells were collected and firefly and Renilla luciferase activities were measured using a dual-luciferase reporter assay (Promega Corporation) according to the manufacturer's protocol. Firefly luciferase activity was normalized to Renilla luciferase activity.

Western blotting. A total of $100 \mu 1$ RIPA cell lysis buffer (cat. no. 78425; Pierce; Thermo Fisher Scientific, Inc.) containing protease inhibitors was added to BT-549 cells on ice for $20 \mathrm{~min}$. Cell lysates were centrifuged at 1,000 x g for $15 \mathrm{~min}$ in a high-speed centrifuge at $4^{\circ} \mathrm{C}$, and supernatants were collected for protein concentration determination using a BCA protein assay kit (Pierce; Thermo Fisher Scientific, Inc.) according to the manufacturer's protocol. Protein samples (40 $\mu \mathrm{g} /$ lane) were loaded on a $10 \%$ SDS-PAGE gel, and then transferred to a PVDF membrane. The membrane 
was blocked with $1 \mathrm{X}$ TBS-0.1\% Tween-20 buffer (TBST) containing $5 \%$ skimmed milk powder for $2 \mathrm{~h}$ at room temperature. Subsequently, the membrane was incubated with the corresponding primary antibodies against SOCS3 (cat. no. ab280884; 1:1,000; Abcam) and GAPDH (cat. no. ab181602; 1:1,000; Abcam) at $4^{\circ} \mathrm{C}$ overnight. After washing with TBST (10X TBS; 20\% Tween-20), the membrane was incubated with the horseradish peroxidase-conjugated secondary goat anti-rabbit IgG antibody (cat. no. ab6721; 1:2,000; Abcam) or goat anti-mouse IgG antibody (cat. no. ab205719; 1:2,000; Abcam) for $1 \mathrm{~h}$ at room temperature. Immunoreactive bands were visualized using enhanced chemiluminescent substrate (Beyotime Institute of Biotechnology). Optical density was measured and relative protein expression was calculated by normalization to GAPDH using ImageJ v1.51 (National Institutes of Health).

MTT assay. Following transfection for 24 h, BT-549 cells were seeded in a $96-$ well plate at a density of $1 \times 10^{4} /$ well and cultured at $37^{\circ} \mathrm{C}$ in a $5 \% \mathrm{CO}_{2}$ incubator. At 24,48 and $72 \mathrm{~h}, 20 \mu \mathrm{l}$ of $5 \mathrm{mg} / \mathrm{ml}$ MTT solution (Beijing Solarbio Science \& Technology Co., Ltd.) were added to each well. The supernatant was subsequently removed after centrifugation $(1,000 \mathrm{x} \mathrm{g} ; 10 \mathrm{~min}$; room temperature). A total of $200 \mu \mathrm{l} \mathrm{DMSO}$ was added to each well, followed by incubation for $30 \mathrm{~min}$ at $37^{\circ} \mathrm{C}$. The plate was then placed in a microplate reader and the optical density was measured at $570 \mathrm{~nm}$. All measurements were repeated in triplicate, and each experiment was repeated at least three times.

Flow cytometry. BT-549 cells were transfected for $48 \mathrm{~h}$ and digested with trypsin (at $4^{\circ} \mathrm{C}$ for $1 \mathrm{~h}$ ) without EDTA and collected. After washing with PBS, the cells were centrifuged at 2,000 rpm for $5 \mathrm{~min}$. The cells were resuspended in $500 \mu \mathrm{l}$ of $1 \mathrm{X}$ PBS solution. $100 \mu \mathrm{l}$ of cell suspension was transferred to a new tube, and $5 \mu \mathrm{l}$ of Annexin V-FITC solution (C1062S) and $5 \mu \mathrm{l}$ of propidium iodide (PI) (both from Beyotime Institute of Biotechnology) solution were added to the cell suspension. Cells were incubated for $5 \mathrm{~min}$ in the dark at room temperature. Detection was performed on a BD FACSCanto II flow cytometer (BD Biosciences) and the data was analyzed using FlowJo software v7.6.1 (FlowJo LLC).

Wound healing assay. BT-549 cells in the logarithmic growth phase ( $24 \mathrm{~h}$ after transfection) were seeded in 24 -well plates at $2 \times 10^{5}$ cells/well and incubated at $37^{\circ} \mathrm{C}$ in a $5 \% \mathrm{CO}_{2}$ incubator to $\sim 100 \%$ confluence. The surface of the cell monolayer in the center of each well was scratched with a $200-\mu 1$ sterile pipette tip. After rinsing with sterile PBS, cells were cultured in serum-free RPMI-1640 medium. The width of the wound was photographed at 0 and $48 \mathrm{~h}$ under a light microscope (magnification, x100; Olympus Corporation).

Transwell assay. A total of $30 \mu \mathrm{l}$ pre-diluted Matrigel $\left(37^{\circ} \mathrm{C}\right.$ for $30 \mathrm{~min}$; BD Biosciences) was placed on top of each Transwell with an $8-\mu \mathrm{m}$ pore size. A total of $24 \mathrm{~h}$ after transfection, BT-549 cells were resuspended in $200 \mu \mathrm{l}$ serum-free RPMI-1640 medium at 5x 105/well and seeded into the upper chamber. In the lower chamber, $700 \mu$ l complete RPMI-1640 medium containing $20 \%$ FBS was added. The cells were then incubated at $37^{\circ} \mathrm{C}$ and $5 \% \mathrm{CO} 2$ for $48 \mathrm{~h}$. Subsequently, the cells in the top chamber were wiped with a cotton swab. The cells invading the lower compartment of the chamber were fixed at room temperature with $4 \%$ methanol for $15 \mathrm{~min}$, and subsequently stained with $0.1 \%$ crystal violet for $10 \mathrm{~min}$ at room temperature. The number of invading cells were counted in five random fields a light microscope at x100 magnification.

Enzyme-linked immunosorbent assay (ELISA). The levels of IL-1 $\beta$ (cat. no. PI305), IL-6 (cat. no. PI330), TNF- $\alpha$ (cat. no. PT518) and MCP-1 (cat. no. PC130) were detected by ELISA (Beyotime Institute of Biotechnology) according to the manufacturer's protocols. Briefly, total proteins were extracted from the BT-549 cells using lysis buffer and the supernatants were collected by centrifuging at $4^{\circ} \mathrm{C}$ at $12,000 \mathrm{x}$ g for $15 \mathrm{~min}$. Subsequently, 96-well microplates were coated with $100 \mu \mathrm{l}$ biotinylated primary antibodies mixed with $100 \mu$ l EIA buffer provided in the kit, plus $100 \mu \mathrm{l}$ standard and sample aliquots. Plates were incubated for $2 \mathrm{~h}$ at $30^{\circ} \mathrm{C}$, followed by aspiration of the samples and subsequent washing of them three times with wash buffer. Next, $100 \mu \mathrm{l}$ solution of streptavidin-horseradish peroxidase conjugate was added to each well and incubated for $30 \mathrm{~min}$ at $30^{\circ} \mathrm{C}$, prior to washed again. Thereafter, $100 \mu \mathrm{l}$ substrate solution provided in the kits was added to each well and the plates were incubated for $30 \mathrm{~min}$ at $30^{\circ} \mathrm{C}$. The optical density values were read at $450 \mathrm{~nm}$ using a Biotek Synergy 2 plate reader (BioTek Instruments, Inc.).

Statistical analysis. Data are presented as the mean \pm standard deviation. Statistical analyses were carried out with SPSS 20.0 (IBM Corp.) and GraphPad Prism v8.0 (GraphPad Software, Inc.). An unpaired two-tailed Student's t-test was used to assess the statistical significance for comparisons between two groups. Comparisons between multiple groups were performed using one-way ANOVA followed by Tukey's post hoc test. In vitro experiments were repeated three times in triplicate. The correlation between miR-483-5p and SOCS3 protein in TNBC tissues was analyzed using Pearson's correlation coefficient analysis. $\mathrm{P}<0.05$ was considered to indicate a statistically significant difference.

\section{Results}

miR-483-5p is upregulated in TNBC tissues and cell lines. The expression level of miR-483-5p in TNBC tissues and adjacent normal tissues was first assessed by RT-qPCR. miR-483-5p expression levels were significantly higher in TNBC tissues compared with adjacent normal tissues (Fig. 1A). Additionally, in the TNBC cell lines BT-20, MDA-MB-231, MDA-MB-468 and BT-549, miR-483-5p expression was also significantly higher compared with in the non-malignant epithelial cell line MCF-10a (Fig. 1B). As the expression level of miR-483-5p was higher in BT-549 cells compared with the other TNBC cell lines, BT-549 was selected for subsequent experiments.

SOCS3 is a target of miR-483-5p. The online software miRTarBase was used to predict the mRNAs putatively targeted by miR-483-5p. A binding site of miR-483-5p was detected in the 3'-UTR of SOCS3 (Fig. 2A). Subsequently, luciferase reporter vectors containing either SOCS3-WT or SOCS3-MUT 3'-UTRs were constructed and co-transfected 

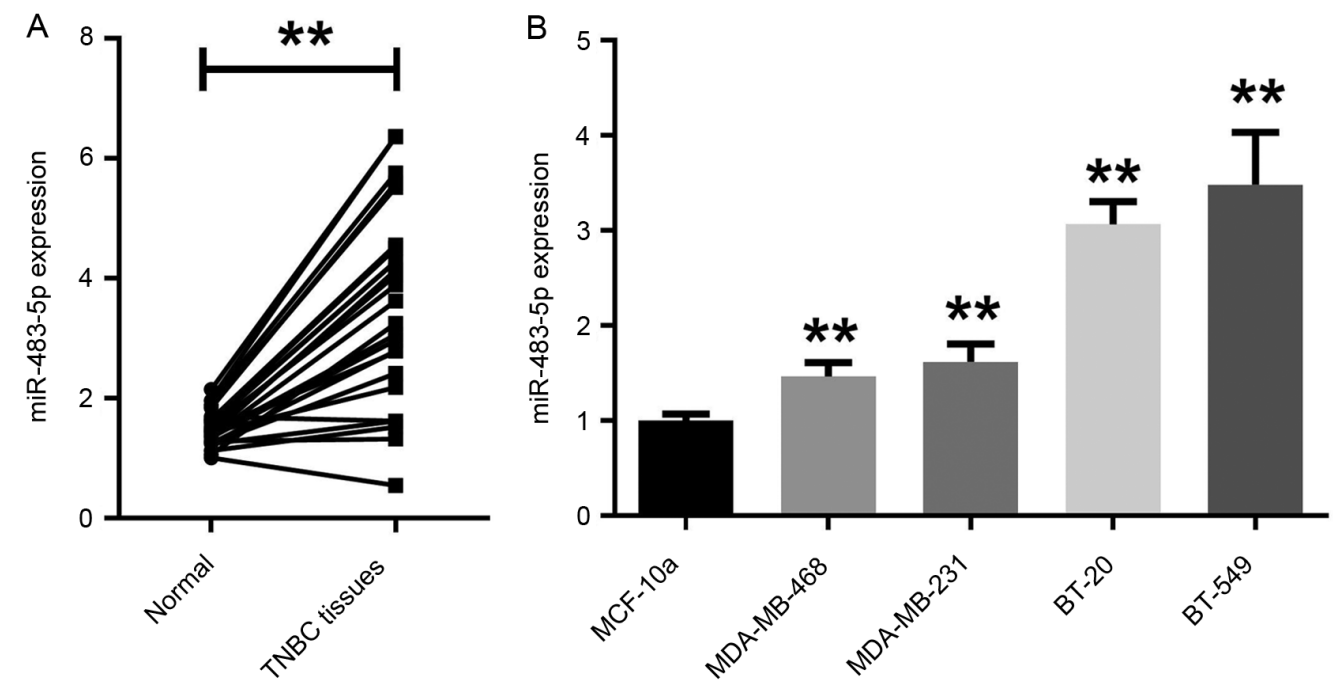

Figure 1. miR-483-5p is upregulated in TNBC tissues and cell lines. (A) Expression of miR-483-53p in TNBC tissues and non-tumor adjacent tissues was detected by RT-qPCR ( $\mathrm{n}=25)$. ${ }^{* *} \mathrm{P}<0.01$. (B) Expression of miR-483-5p in TNBC cell lines (BT-20, MDA-MB-231, MDA-MB-468, BT-549) and the non-malignant breast epithelial cell line MCF-10a was analyzed by RT-qPCR. Data are presented as the mean \pm SD of three independent experiments. ${ }^{* *} \mathrm{P}<0.01$ vs. MCF-10a cells. RT-qPCR, reverse transcription-quantitative PCR; TNBC, triple-negative breast cancer; miR, microRNA.

A

SOCS3-Wt 5' tcCCCTTCCTCCACACCCCCTCTg 3'

miR-483-5p 3' gaGGGAA-GAGAAG-AGGGCAGAa 5'

SOCS3-Mut 5' tcGGGAACGAGCCAACGGGCAGAg 3'
B

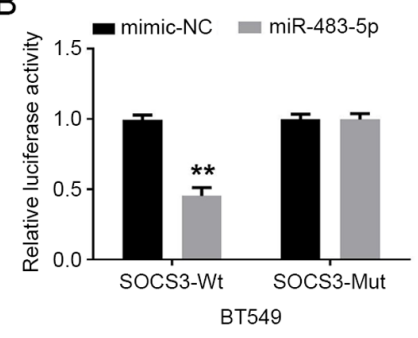

$E$

m inhibitor-NC miR-483-5p inhibitor mimic-NC miR-483-5p-mimic

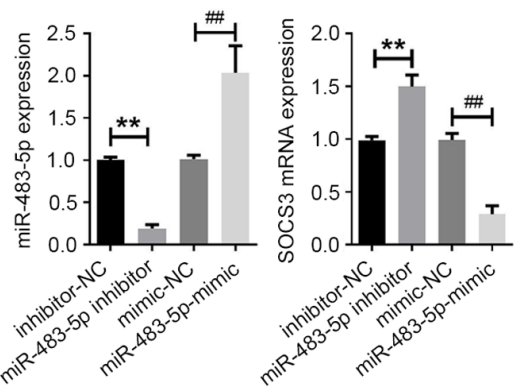

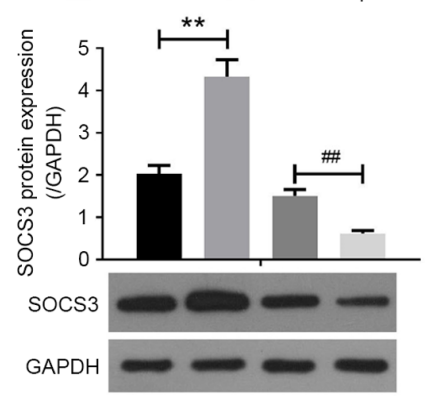

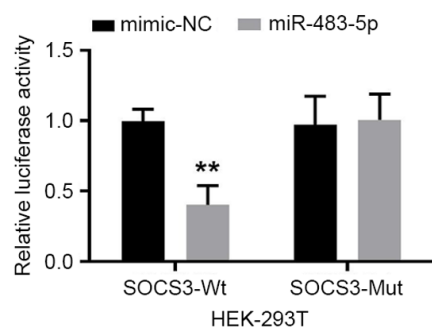

$\mathrm{F}$
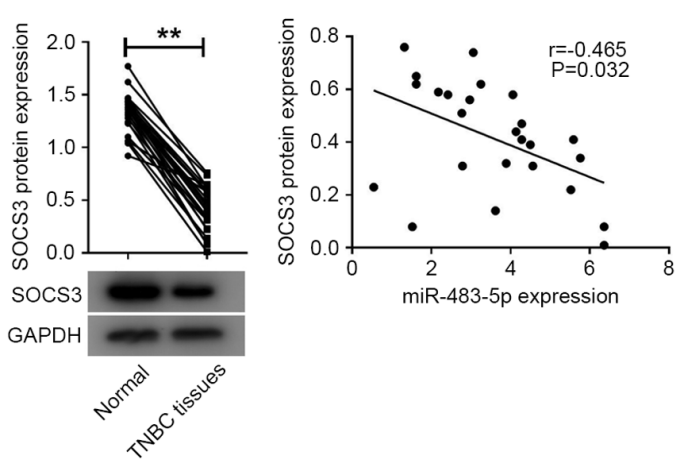

Figure 2. SOCS3 is a target of miR-483-5p. (A) miR-483-5p binding site was predicted in the 3'-UTR of SOCS3 mRNA. (B) Luciferase reporter vectors containing WT or MUT SOCS3 3'-UTR were co-transfected with miR-483-5p mimics or mimic-NC into BT-549 and 293T cells, and a luciferase reporter assay was performed. ${ }^{* *} \mathrm{P}<0.01$ vs. SOCS3-WT + mimic-NC. (C) Expression of miR-483-5p and SOCS3 mRNA in BT-549 cells was analyzed via reverse transcription-quantitative PCR. ${ }^{* *} \mathrm{P}<0.01 ;{ }^{\# \#} \mathrm{P}<0.01$. (D) SOCS3 protein expression was detected via western blotting in $\mathrm{BT}-549$ cells transfected with miR-483-5p mimics or inhibitor. ${ }^{* *} \mathrm{P}<0.01$; ${ }^{\# \#} \mathrm{P}<0.01$. (E) Expression of SOCS3 protein in TNBC tissues was examined via western blotting. ${ }^{* *} \mathrm{P}<0.01$. (F) Correlation analysis between miR-483-5p and SOCS3 expression in TNBC tissues. Data are presented as the mean \pm SD of three independent experiments. 3'-UTR, 3'-untranslated region; TNBC, triple-negative breast cancer; miR, microRNA; NC, negative control; SOCS3, suppressor of cytokine signaling 3; Wt, wild-type; Mut, mutant.

with miR-483-5p mimics or mimic-NC into BT-549 and 293T cells to perform luciferase reporter assays. Co-transfection with miR-483-5p mimics was observed to significantly reduce the luciferase activity of the SOCS3-WT vector, while the luciferase activity of the reporter containing SOCS3-MUT was unaffected in cells following transfection with miR-483-5p mimics compared with mimic-NC (Fig. 2B).
Additionally, miR-483-5p inhibitor or miR-483-5p mimics were transfected into BT-549 cells. The expression levels of miR-483-5p, SOCS3 mRNA and SOCS3 protein were quantified by RT-qPCR and western blot assay. RT-qPCR analysis indicated that miR-483-5p inhibitor decreased miR-483-5p expression in BT-549 cells, while miR-483-5p mimics exhibited the opposite effect compared with the respective NC (Fig. 2C). 
Moreover, compared with inhibitor-NC, miR-483-5p inhibitor markedly increased the expression level of SOCS3 mRNA and protein in BT-549 cells. By contrast, compared with mimic-NC, miR-483-5p mimics significantly reduced SOCS3 mRNA and protein expression levels in BT-549 cells (Fig. 2C and D).

Detection of SOCS3 protein in TNBC tissues via western blot analysis indicated that the expression level of SOCS3 protein in TNBC tissues was significantly downregulated compared with in adjacent normal tissues (Fig. 2E). Correlation analysis revealed that miR-483-5p expression was negatively correlated with SOCS3 protein expression in TNBC tissues (Fig. 2F). Taken together, these results indicated that SOCS3 was one of the targets of miR-483-5p, and that miR-483-5p could regulate the expression of SOCS3 in TNBC cells.

Knockdown of miR-483-5p inhibits the proliferation and promotes apoptosis of the TNBC cell line BT-549 cells by regulating SOCS3. To investigate whether miR-483-5p could participate in TNBC progression by regulating SOCS3, miR-483-5p inhibitor and si-SOCS3 were co-transfected into BT-549 cells. As illustrated in Fig. 3A, transfection with si-SOCS3 successfully decreased the expression level of SOCS3 in BT-549 cells compared with the control siRNA. Western blot assay results indicated that miR-483-5p inhibitor promoted the expression of SOCS3, which could be reversed by si-SOCS3 (Fig. 3B). The effects of miR-483-5p on the proliferation and apoptosis of BT-549 cells were further evaluated by MTT assays and flow cytometry. As depicted in Fig. 3C, the decrease of miR-483-5p inhibited the proliferation of BT-549 cells, which was markedly attenuated by si-SOCS3 transfection. Additionally, flow cytometry revealed that the inhibition of miR-483-5p significantly increased apoptosis in BT-549 cells, which was reversed by si-SOCS3 (Fig. 3D).

miR-483-5p mimics and SOCS3-OE were then co-transfected into BT-549 cells. The expression level of SOCS3 was increased in BT-549 cells transfected with SOCS3-OE compared with the empty vector (Fig. 3E). SOCS3-OE attenuated the inhibitory effect of miR-483-5p on SOCS3 expression in BT-549 cells (Fig. 3F). SOCS3-OE also partially suppressed the promoting effect of miR-483-5p on the proliferation of BT-549 cells (Fig. 3G). Consistently, SOCS3 overexpression attenuated the miR-483-5p-mediated inhibition of apoptosis (Fig. 3H). These data suggested that knockdown of miR-483-5p could inhibit the proliferation of BT-549 cells and induce apoptosis by targeting SOCS3.

Knockdown of miR-483-5p inhibits the migration and invasion of the TNBC cell line BT-549 cells by regulating SOCS3. Metastasis is the leading cause of death in clinical patients with breast cancer (17). The effects of miR-483-5p on BT-549 migration and invasion were investigated. Wound healing assay results indicated that the decrease of miR-483-5p inhibited BT-549 cell migration, which was markedly reversed by SOCS3 siRNA transfection (Fig. 4A). Consistent with this observation, Transwell assays revealed that SOCS3 siRNA reversed the inhibitory effect of miR-483-5p inhibitor on BT-549 cell invasion (Fig. 4B). Additionally, the effect of miR-483-5p mimics on the migration (Fig. 4C) and invasion (Fig. 4D) of BT-549 cells was reversed by SOCS3-OE transfection.
Knockdown of miR-483-5p reduces the secretion of inflammatory factors in TNBC cells by regulating SOCS3. In addition to metastasis, the occurrence of inflammation is an important factor in the worsening of breast cancer. It has been indicated that inflammatory factors, such as TNF- $\alpha$, IL-6, IL-1 $\beta$ and monocyte chemoattractant protein-1 (MCP-1), are closely associated with the recurrence and metastasis of breast cancer (18). Therefore, TNF- $\alpha$, IL- 6 , IL-1 $\beta$ and MCP-1 were quantified in BT-549 cell supernatant via ELISA. Compared with the inhibitor-NC group, miR-483-5p inhibitor notably reduced the secretion of IL-1 $\beta$, IL- 6 , TNF- $\alpha$ and MCP-1 in BT-549 cells, while si-SOCS3 partially reversed the effect of miR-483-5p inhibitor on the inflammatory response (Fig. 5A-D). On the contrary, increases in inflammatory factor secretion induced by miR-483-5p mimics in BT-549 cells was reversed after transfection with SOCS3-OE (Fig. 5E-H).

\section{Discussion}

miRNAs have been indicated to play roles similar to oncogenes or tumor suppressors (4). In the present study, the expression level of miR-483-5p was observed to be increased in TNBC tissues and cell lines compared with normal adjacent tissues and cells, and the inhibition of miR-483-5p could inhibit the proliferation, invasion and inflammatory response of TNBC cells. More importantly, SOCS3 was one of the targets of miR-483-5p, and SOCS3 silencing reversed the anti-oncogenic effect of miR-483-5p knockdown on TNBC cells.

In the present study, miR-483-5p was identified as a novel therapeutic target for TNBC. Upregulation of miR-483-5p expression has previously been reported in adrenal cortical cancer and esophageal squamous cell carcinoma $(14,19)$. Consistent with these reports, the present study provided new evidence for the abnormal expression of miR-483-5p in TNBC. Interestingly, Wang et al (20) reported that miR-483-5p is decreased in human glioma. Considering that gene expression is tumor-specific and that the reason for the differential expression of miRNAs depends to a large extent on the condition of the tumor $(21,22)$, these results are not contradictory. miR-483-5p was inhibited in the TNBC cell line BT-549 to analyze cell proliferation and invasion. The results indicated that inhibition of miR-483-5p expression inhibited the proliferative and invasive capabilities of BT-549 cells. In addition, the anti-proliferative effect of miR-483-5p silencing may be related to its positive effect on apoptosis. As the interaction between the tumor microenvironment and tumor cells has received increasing attention, tumor-associated inflammation is also considered to be an important feature of cancers. For instance, inflammation has been revealed to be closely associated with the growth, angiogenesis and distant metastasis of breast tumor cells (23). Moreover, excessive inflammation can disrupt the acquired immune response and reduce the sensitivity of cells to chemotherapeutics (24). Furthermore, blocking pro-inflammatory cytokines can be used as a therapeutic strategy for targeting TNBC (25). In the present study, inhibition of miR-483-5p reduced the secretion of inflammatory cytokines in breast cancer cells. These results indicated that miR-483-5p may be a potential oncogenic factor for the progression of TNBC. 
A

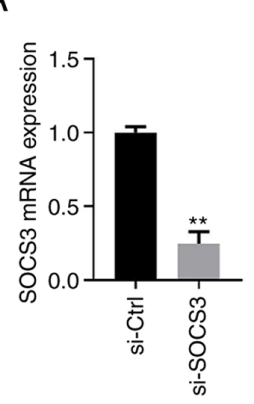

D

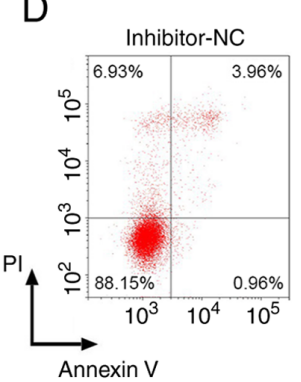

$\mathrm{E}$

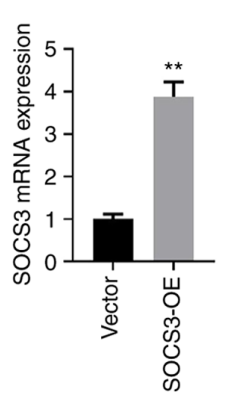

$\mathrm{H}$

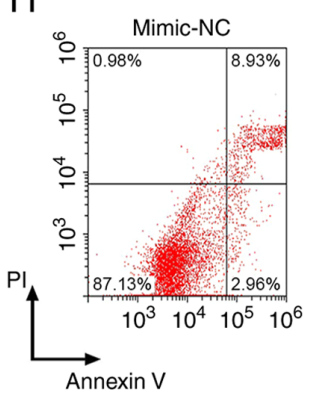

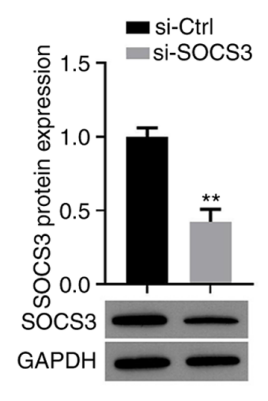

B
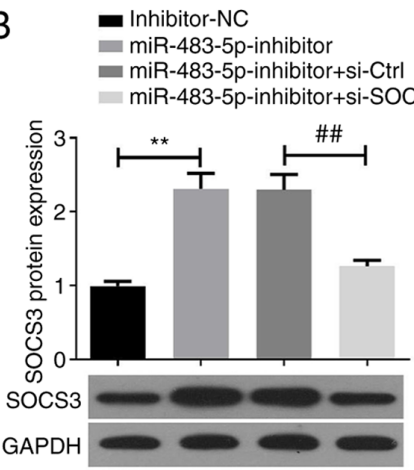

C Inhibitor-NC

- miR-483-5p-inhibitor

- miR-483-5p-inhibitor+si-Ctrl

- miR-483-5p-inhibitor+si-SOCS3

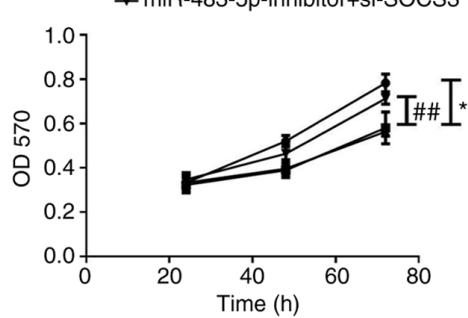

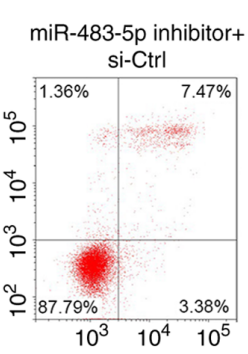

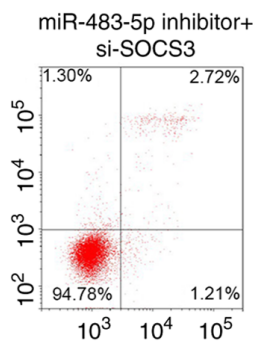

$\mathrm{F}$
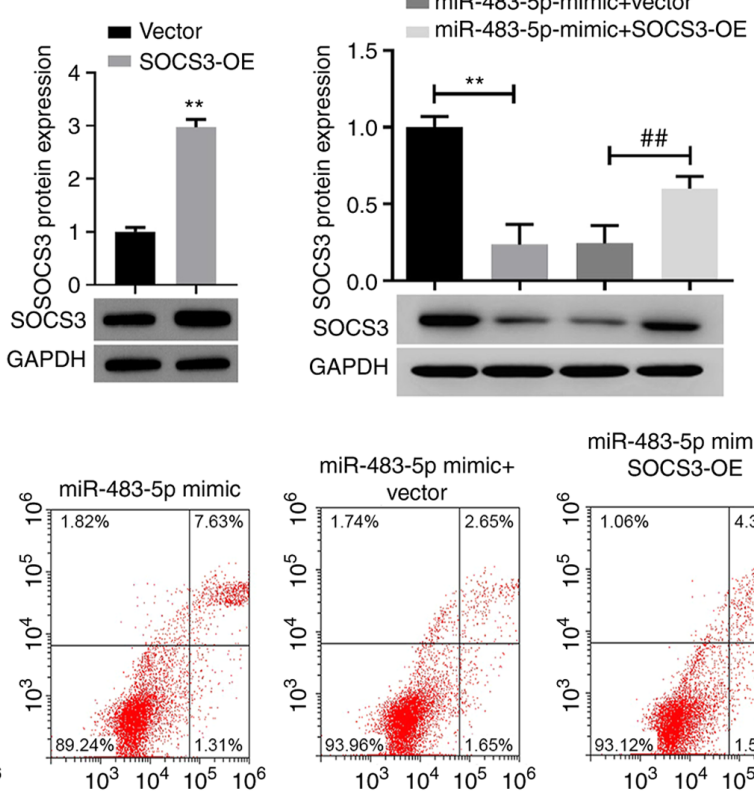

miR-483-5p mimic SOCS3-OE
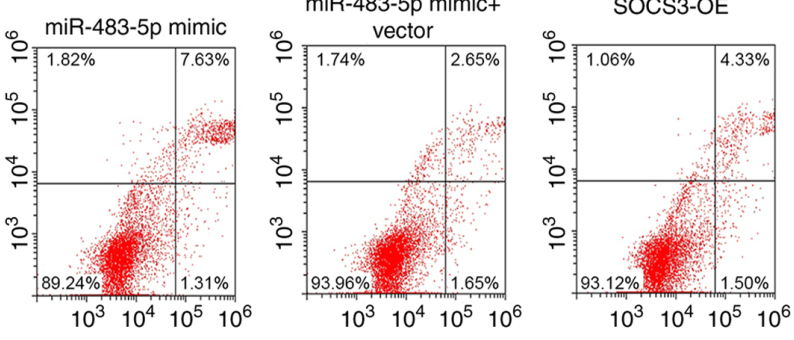

G

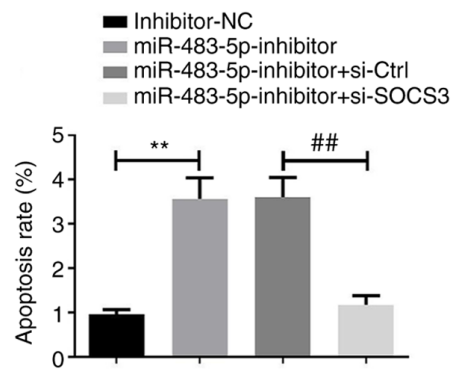

$\rightarrow$ Mimic-NC

- miR-483-5p-mimic

- miR-483-5p-mimic+vector

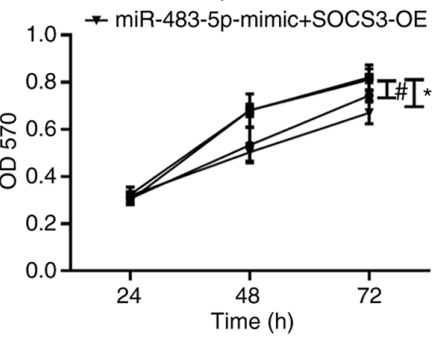

- Mimic-NC

miR-483-5p-mimic

- miR-483-5p-mimic+vector

$=$ miR-483-5p-mimic+SOCS3-OE

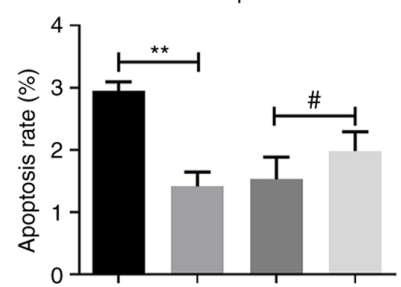

Figure 3. miR-483-5p knockdown inhibits the proliferation and promotes apoptosis of BT-549 cells by regulating SOCS3. (A) Expression of SOCS3 mRNA and protein in BT-549 cells transfected with si-Ctrl or si-SOCS3 was analyzed using RT-qPCR or western blot assay. ${ }^{* *}$ P $<0.01$ vs. si-Ctrl. (B) SOCS3 protein expression was analyzed via western blotting, (C) cell proliferation was detected using MTT assay and (D) flow cytometry was used to determine apoptosis in BT-549 cells co-transfected with miR-483-5p inhibitor and si-SOCS3. ${ }^{* *} \mathrm{P}<0.01 ;{ }^{\# \#} \mathrm{P}<0.01$. (E) Expression of SOCS3 mRNA and protein in BT-549 cells transfected with vector or SOCS3-OE was analyzed using RT-qPCR or western blot assays. ${ }^{* *} \mathrm{P}<0.01$ vs. Vector. (F) SOCS3 protein expression was analyzed via western blotting in BT-549 cells co-transfected with miR-483-5p mimic and SOCS3-OE. (G) Proliferation and (H) apoptosis of BT-549 cells co-transfected with miR-483-5p mimic and SOCS3-OE was analyzed using MTT assays and flow cytometry, respectively. Data are presented as the mean \pm SD of three independent experiments. $\mathrm{P}<0.05,{ }^{* *} \mathrm{P}<0.01 ;{ }^{\#} \mathrm{P}<0.05,{ }^{\# \#} \mathrm{P}<0.01$. RT-qPCR, reverse transcription-quantitative PCR; miR, microRNA; NC, negative control; SOCS3, suppressor of cytokine signaling 3; Ctrl, control; si, small interfering; OE, overexpression; OD, optical density; PI, propidium iodide.

It is well known that miRNAs can perform biological functions by regulating the expression of multiple target genes (4). In the current study, miRTarBase analysis revealed that SOCS3 was one of the direct targets of miR-483-5p. Luciferase reporter assays and expression level analysis confirmed that
miR-483-5p was able to negatively regulate SOCS3 expression in BT-549 cells. SOCS3 is a cytokine signaling inhibitor protein, and its abnormal expression has been associated with the occurrence and development of numerous diseases, including tumors (26). It has been previously demonstrated 
A

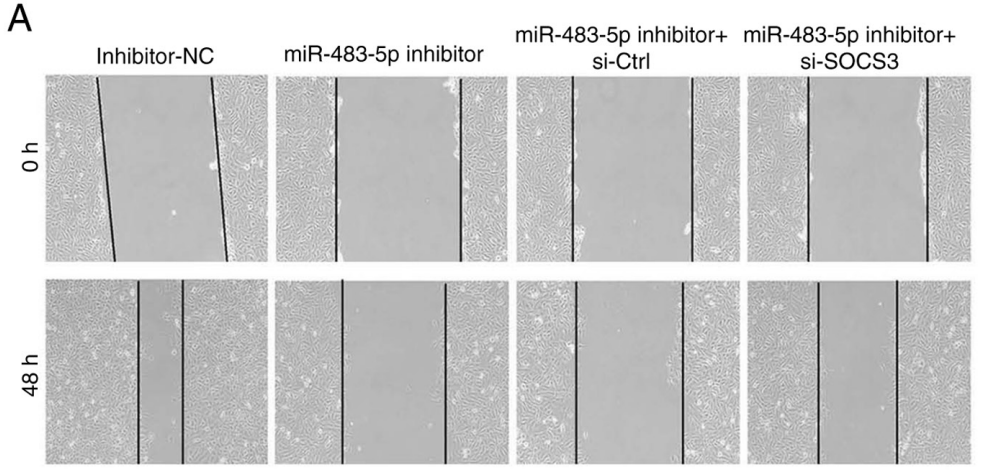

B

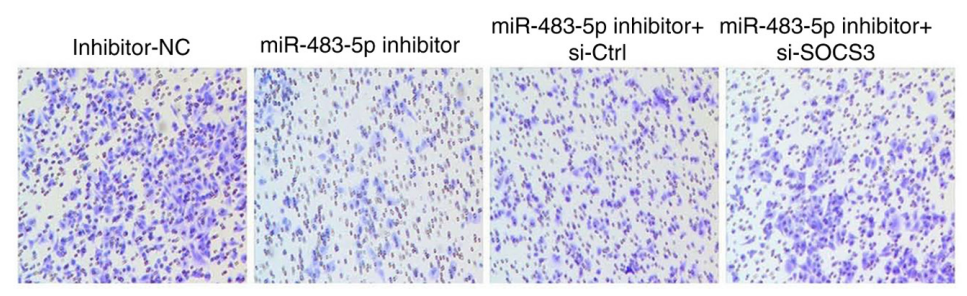

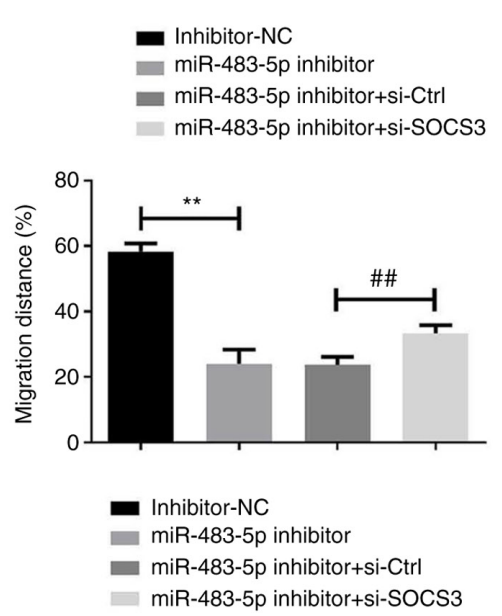

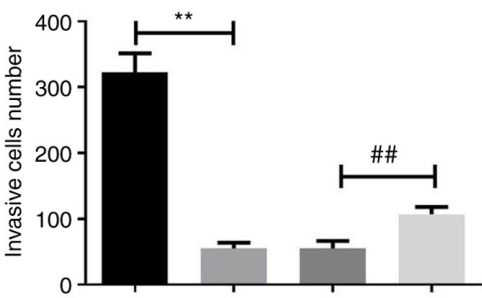

- Mimic-NC

miR-483-5p-mimic

miR-483-5p-mimic+vector

miR-483-5p-mimic+SOCS3-OE

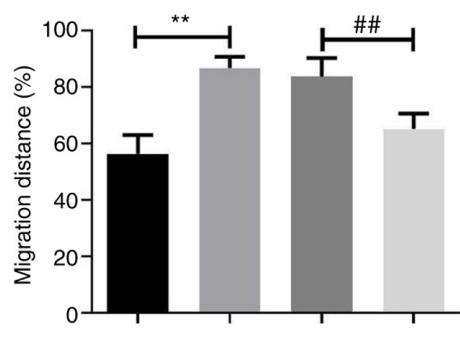

- Mimic-NC

- miR-483-5p-mimic

miR-483-5p-mimic+vecto

- miR-483-5p-mimic+SOCS3-OE

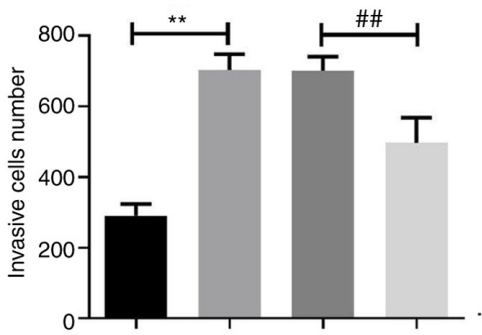

Figure 4. miR-483-5p knockdown inhibits migration and invasion of the triple-negative breast cancer cell line BT-549 by regulating SOCS3. (A) Wound healing assay was used to analyze the migration of BT-549 cells co-transfected with miR-483-5p inhibitor and si-SOCS3. Scale bar, 200 $\mu$ m. (B) Transwell assay was used to analyze the invasion of BT-549 cells co-transfected with miR-483-5p inhibitor and si-SOCS3. Scale bar, $100 \mu \mathrm{m} .{ }^{* *} \mathrm{P}<0.01$; ${ }^{\# \#} \mathrm{P}<0.01$. (C) Wound healing assay was used to analyze the migration of BT-549 cells co-transfected with miR-483-5p mimic and SOCS3-OE. Scale bar, 200 $\mu \mathrm{m}$. (D) Transwell assay was used to analyze the invasion of BT-549 cells co-transfected with miR-483-5p mimic and SOCS3-OE. Scale bar, 100 $\mu$ m. Data are presented as the mean \pm SD of three independent experiments. ${ }^{* *} \mathrm{P}<0.01$; ${ }^{\# \#} \mathrm{P}<0.01$. miR, microRNA; NC, negative control; SOCS3, suppressor of cytokine signaling 3; Ctrl, control; si, small interfering; OE, overexpression.

that SOCS3 can regulate the proliferation, metastasis and invasion of several types of tumor cells (27-29), including breast cancer (30). Furthermore, it has been indicated that the expression of the SOCS3 gene is directly associated with the expression of several inflammatory genes, especially NF- $\mathrm{kB}$, primary inflammatory cytokines (such as TNF- $\alpha$, 
A

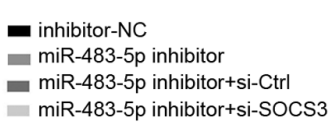

B

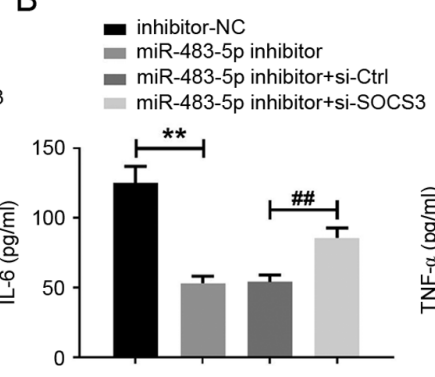

C

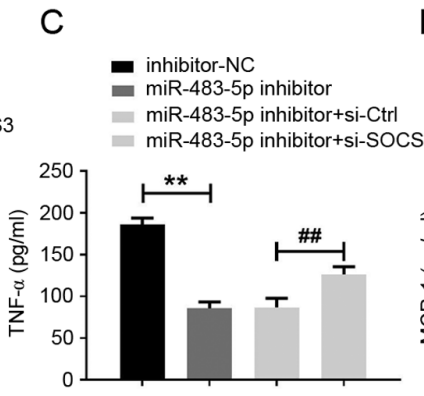

D

$$
\begin{aligned}
& \text { inhibitor-NC } \\
& \text { miR-483-5p inhibitor } \\
& \text { miR-483-5p inhibitor+si-Ctrl } \\
& \text { miR-483-5p inhibitor+si-SOCS3 }
\end{aligned}
$$

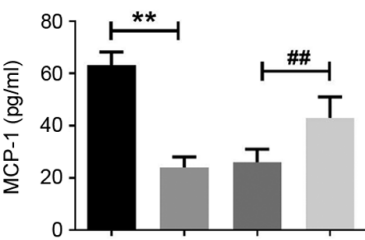

E
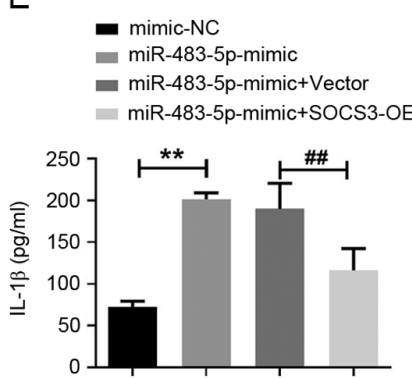

$\mathrm{F}$

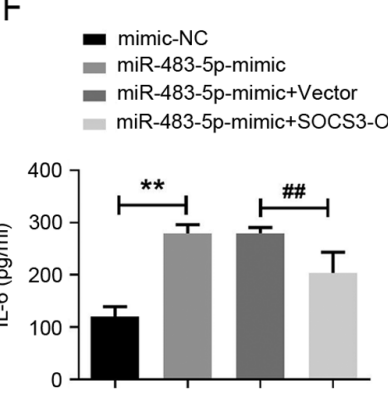

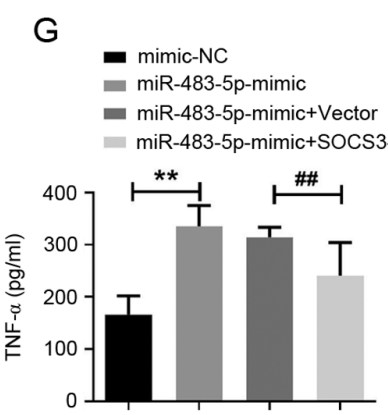

$\mathrm{H}$

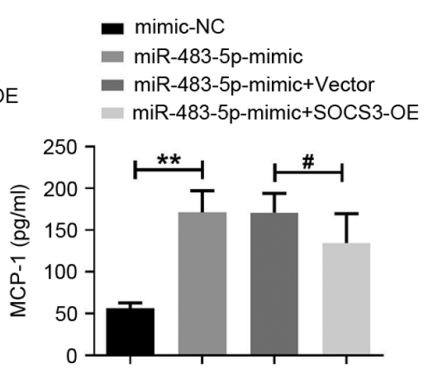

Figure 5. Knockdown of miR-483-5p reduces the secretion of inflammatory factors in triple-negative breast cancer cells by regulating SOCS3. ELISA was used to analyze the quantity of (A) IL-1 $\beta$, (B) IL-6, (C) TNF- $\alpha$ and (D) MCP-1 in the supernatant of BT-549 cells co-transfected with miR-483-5p inhibitor and si-SOCS3. ${ }^{* *} \mathrm{P}<0.01 ;{ }^{\# /} \mathrm{P}<0.01$. ELISA was used to analyze the quantity of (E) IL-1 $\beta$, (F) IL-6, (G) TNF- $\alpha$ and (H) MCP-1 in the supernatant of BT-549 cells co-transfected with miR-483-5p mimic and SOCS3-OE. ${ }^{* *} \mathrm{P}<0.01 ;{ }^{\#} \mathrm{P}<0.05,{ }^{\# \#} \mathrm{P}<0.01$. Data are presented as the mean \pm SD of three independent experiments. MCP-1, monocyte chemoattractant protein-1; miR, microRNA; NC, negative control; SOCS3, suppressor of cytokine signaling 3; Ctrl, control; si,small interfering; OE, overexpression.

IL-6, IL-1 $\beta$ ) and the STAT3 cascade (31), which have been considered as the main molecular participants involved in cancer-related inflammation $(32,33)$. It has also been demonstrated in TNBC that enhanced expression of SOCS3 can inhibit tumor growth and metastasis by interfering with the IL-6/STAT3/NF- $\kappa$ B pathway (21). In the present study, miR-483-5p inhibitor and si-SOCS3 were co-transfected into BT-549 cells, and the results indicated that SOCS3 silencing reversed the effects of miR-483-5p inhibition on cell proliferation, apoptosis, migration, invasion and inflammatory factor secretion. Additionally, co-transfection of miR-483-5p and SOCS3-OE also abolished the effects of miR-483-5p alone on the malignant phenotype of TNBC cells. Based on these results, it could be speculated that miR-483-5p may exert a carcinogenic role in TNBC by directly inhibiting the expression of SOCS3.

However, there were certain limitations in the present study. Firstly, the sample size of patients with TNBC was limited. Moreover, it was only confirmed that SOCS3 was one of the targets of miR-483-5p. It is well known that miRNAs can target and regulate a variety of genes (34). Therefore, future studies may involve an extensive screening of more targets through microarrays to further clarify the potential mechanism of miR-483-5p in the progression of TNBC.

In conclusion, the expression level of miR-483-5p was revealed to be increased in TNBC tissues and cell lines, and inhibition of miR-483-5p inhibited the proliferation, migration, invasion and inflammatory response, while promoting apoptosis of TNBC cells by negatively regulating SOCS3. Therefore, miR-483-5p may be a potential target for TNBC therapy.

\section{Acknowledgements}

Not applicable.

\section{Funding}

No funding was received.

\section{Availability of data and materials}

All data generated or analyzed during this study are included in this published article.

\section{Authors' contributions}

JR and GX wrote the manuscript and conducted the majority of the experiments. HS and TL analyzed data and conducted the experiments. SX acquired data and performed statistical analysis. YZ designed the research, analyzed the data and approved the study. JR and YZ confirm the authenticity of all the raw data. All authors read and approved the final manuscript.

\section{Ethics approval and consent to participate}

The present study was approved by Yantai Muping Hospital of Traditional Chinese Medicine (Yantai, China), and written informed consent was obtained from all the participants.

\section{Patient consent for publication}

Not applicable. 


\section{Competing interests}

The authors declare that they have no competing interests.

\section{References}

1. Domínguez F, Maycotte P, Acosta-Casique A, RodríguezRodríguez S, Moreno DA, Ferreres F, Flores-Alonso JC, Delgado-López MG, Pérez-Santos M and Anaya-Ruiz M: Bursera copallifera Extracts have cytotoxic and migration-inhibitory effects in breast cancer cell lines. Integr Cancer Ther 17: 654-664, 2018.

2. Geenen JJJ, Linn SC, Beijnen JH and Schellens JH: PARP inhibitors in the treatment of triple-negative breast cancer. Clin Pharmacokinet 57: 427-437, 2018.

3. Liu J, Shi Z, Bai Y, Liu L and Cheng K: Prognostic significance of systemic immune-inflammation index in triple-negative breast cancer. Cancer Manag Res 11: 4471-4480, 2019.

4. Paul S, Reyes PR, Garza BS and Sharma A: MicroRNAs and child neuropsychiatric disorders: A Brief Review. Neurochem Res 45: 232-40, 2020.

5. Dai W, Lu H, Yang F, Dong H and Zhang X: Accurate detection of intracellular microRNAs using functional Mo2C quantum dots nanoprobe. Chem Commun (Camb) 55: 10615-10618, 2019.

6. Li H, He C, Wang X, Wang H, Nan G and Fang L: MicroRNA-183 affects the development of gastric cancer by regulating autophagy via MALAT1-miR-183-SIRT1 axis and PI3K/AKT/mTOR signals. Artif Cells Nanomed Biotechnol 47: 3163-3171, 2019.

7. Lou Q, Liu R, Yang X, Li W, Huang L, Wei L, Tan H, Xiang N, Chan K, Chen J, et al: miR-448 targets IDO1 and regulates CD8 $\mathrm{T}$ cell response in human colon cancer. J Immunother Cancer 7 : 210, 2019.

8. Ma L, Teruya-Feldstein J and Weinberg RA: Tumour invasion and metastasis initiated by microRNA-10b in breast cancer. Nature 449: 682-688, 2007.

9. Sahraei M, Chaube B, Liu Y, Sun J, Kaplan A, Price NL, Ding W, Oyaghire S, García-Milian R, Mehta S, et al: Suppressing miR-21 activity in tumor-associated macrophages promotes an antitumor immune response. J Clin Invest 129: 5518-5536, 2019.

10. Li H, Bian C, Liao L, Li J and Zhao RC: miR-17-5p promotes human breast cancer cell migration and invasion through suppression of HBP1. Breast Cancer Res Treat 126: 565-575, 2011.

11. Reinhart BJ, Slack FJ, Basson M, Pasquinelli AE, Bettinger JC, Rougvie AE, Horvitz HR and Ruvkun G: The 21-nucleotide let-7 RNA regulates developmental timing in Caenorhabditis elegans. Nature 403: 901-906, 2000.

12. Gao Y, Zhang W, Liu C and Li G: miR-200 affects tamoxifen resistance in breast cancer cells through regulation of MYB. Sci Rep 9: 18844, 2019.

13. Girardot M, Cavaillé J and Feil R: Small regulatory RNAs controlled by genomic imprinting and their contribution to human disease. Epigenetics 7: 1341-1348, 2012.

14. Soon PS, Tacon LJ, Gill AJ, Bambach CP, Sywak MS, Campbell PR, Yeh MW, Wong SG, Clifton-Bligh RJ, Robinson BG, et al: miR-195 and miR-483-5p identified as predictors of poor prognosis in adrenocortical cancer. Clin Cancer Res 15: 7684-7692, 2009.

15. Song Q, Xu Y, Yang C, Chen Z, Jia C, Chen J, Zhang Y, Lai P, Fan $\mathrm{X}$, Zhou X, et al: miR-483-5p promotes invasion and metastasis of lung adenocarcinoma by targeting RhoGDI1 and ALCAM. Cancer Res 74: 3031-3042, 2014.

16. Livak KJ and Schmittgen TD: Analysis of relative gene expression data using real-time quantitative PCR and the 2(-Delta Delta C(T)) method. Methods 25: 402-408, 2001.

17. Meirson T and Gil-Henn H: Targeting invadopodia for blocking breast cancer metastasis. Drug Resist Updat 39: 1-17, 2018

18. De Sanctis V, Agolli L, Visco V, Monaco F, Muni R, Spagnoli A, Campanella B, Valeriani M, Minniti G, Osti MF, et al: Cytokines, fatigue, and cutaneous erythema in early stage breast cancer patients receiving adjuvant radiation therapy. Biomed Res Int 2014: 523568, 2014.
19. Zhang H, Shi X, Chang W, Li Y, Wang L and Wang L: Epigenetic alterations of the Igf 2 promoter and the effect of miR $4835 \mathrm{p}$ on its target gene expression in esophageal squamous cell carcinoma. Mol Med Rep 17: 2251-2256, 2018.

20. Wang L, Shi M, Hou S, Ding B, Liu L, Ji X, Zhang J and Deng Y: MiR-483-5p suppresses the proliferation of glioma cells via directly targeting ERK1. FEBS Lett 586: 1312-1317, 2012.

21. Lemberger M, Loewenstein S, Lubezky N, Nizri E, Pasmanik-Chor M, Barazovsky E, Klausner JM and Lahat G: MicroRNA profiling of pancreatic ductal adenocarcinoma (PDAC) reveals signature expression related to lymph node metastasis. Oncotarget 10: 2644-2656, 2019.

22. Blenkiron C, Goldstein LD, Thorne NP, Spiteri I, Chin SF, Dunning MJ, Barbosa-Morais NL, Teschendorff AE, Green AR, Ellis IO, et al: MicroRNA expression profiling of human breast cancer identifies new markers of tumor subtype. Genome Biol 8: R214, 2007.

23. Conteduca V, Caffo O, Galli L, Maugeri A, Scarpi E, Maines F, Chiuri VE, Cristian Lolli C, Kinspergher S, Schepisi G, et al: Association among metabolic syndrome, inflammation, and survival in prostate cancer. Urol Oncol 36: 240.e1-.e11, 2018.

24. Garbers C and Rose-John S: Dissecting interleukin-6 classic-and trans-signaling in inflammation and cancer. Methods Mol Biol 1725: 127-140, 2018.

25. Kim G, Ouzounova M, Quraishi AA, Davis A, Tawakkol N, Clouthier SG, Malik F, Paulson AK, D'Angelo RC, Korkaya S, et al: SOCS3-mediated regulation of inflammatory cytokines in PTEN and p53 inactivated triple negative breast cancer model. Oncogene 34: 671-680, 2015

26. Klepsch O, Namer LS, Köhler N, Kaempfer R, Dittrich A and Schaper F: Intragenic regulation of SOCS3 isoforms. Cell Commun Signal 17: 70, 2019

27. Li H, Zhang B, Ding M, Lu S, Zhou H, Sun D, Wu G and Gan X: C1QTNF1-AS1 regulates the occurrence and development of hepatocellular carcinoma by regulating miR-221-3p/SOCS3. Hepatol Int 13: 277-292, 2019.

28. Singh S, Chouhan S, Mohammad N and Bhat MK: Resistin causes G1 arrest in colon cancer cells through upregulation of SOCS3. FEBS Lett 591: 1371-1382, 2017.

29. Li MZ, Lai DH, Zhao HB, Chen Z, Huang QX and Situ J: SOCS3 overexpression enhances ADM resistance in bladder cancer T24 cells. Eur Rev Med Pharmacol Sci 21: 3005-3011, 2017.

30. Muhammad N, Bhattacharya S, Steele R and Ray RB Anti-miR-203 suppresses ER-positive breast cancer growth and stemness by targeting SOCS3. Oncotarget 7: 58595-58605, 2016

31. Christopher AF, Gupta M and Bansal P: Micronome revealed $\mathrm{miR}-19 \mathrm{a} / \mathrm{b}$ as key regulator of SOCS3 during cancer related inflammation of oral squamous cell carcinoma. Gene 594: 30-40, 2016.

32. Zakaria N, Mohd Yusoff N,Zakaria Z, Widera D and Yahaya BH: Inhibition of NF- $\mathrm{NB}$ signaling reduces the stemness characteristics of lung ccancer stem cells. Front Oncol 8: 166, 2018.

33. Zhang X, Wan Q and Xu TS: Effect of Grain-moxibustion on IL-6 and STAT 3 in inflammatory microenvironment of Lewis lung cancer mice. Zhen Ci Yan Jiu 42: 235-239, 2017 (In Chinese).

34. Chen L, Heikkinen L, Wang C, Yang Y, Sun H and Wong G: Trends in the development of miRNA bioinformatics tools. Brief Bioinform 20: 1836-1852, 2019.

This work is licensed under a Creative Commons Attribution-NonCommercial-NoDerivatives 4.0 International (CC BY-NC-ND 4.0) License. 